\title{
Modeling High-Pressure Wax Formation in Petroleum Fluids
}

\author{
Jean-Marc Sansot, Jérôme Pauly, and Jean-Luc Daridon \\ Laboratoire des Fluides Complexes-Groupe Haute Pression, Université de Pau, BP 1155, 64013 Pau Cedex, France
}

Joao A. P. Coutinho

Depto. de Química, CICECO, Universidade de Aveiro, 3810-193 Aveiro, Portugal

DOI 10.1002/aic.10434

Published online May 16, 2005 in Wiley InterScience (www.interscience.wiley.com).

\begin{abstract}
A procedure is presented for the simultaneous prediction of fluid-fluid and solid-fluid equilibrium of light gases/heavy hydrocarbons systems under pressure. The objective of this work is to simplify the predictive approach previously proposed by the authors, making it more useful and accessible to users at the petroleum industry. The main modification with respect to the complex LCVM (linear combination of Huron-Vial and Michelsen) mixing rule that was previously used for the attractive term " $a$ " of the equation of state (EOS). To avoid the use of a group contribution method, which is difficult to bring into play with real fluids, the LCVM mixing rule is here replaced by the classical quadratic van der Walls one-fluid mixing rule. The deviation that using this mixing rule introduces into the gamma-phi approach is cancelled by a new parameter $\xi$, introduced in the calculation of the solid-phase activity coefficient. This procedure was tested for a large range of multicomponent systems where the parameter $\xi$ was fitted to match the wax appearance temperature (WAT) at atmospheric pressure. From the $\xi$ values obtained a correlation relating the parameter $\xi$ to the carbon number in the heavy fraction was developed, allowing a prediction of wax formation in petroleum fluids. It is shown that this approach can successfully be applied to the prediction of wax formation at high pressure for live oils. () 2005 American Institute of Chemical Engineers AIChE J, 51: 2089-2097, 2005 Keywords: solid-fluid equilibrium, wax, petroleum, high-pressure, wax appearance temperature (WAT)
\end{abstract}

\section{Introduction}

A major problem in oil extraction and transport is the buildup of paraffin deposits that restrict flow in the pipeline. The solubility of high molecular weight paraffins in aromaticor aliphatic-base oil solvents decreases rapidly with temperature and is further enhanced by pressure. Thus, when waxy oils flow through cooled pipelines, the wax precipitates-creating deposit layers that increase the pressure drop, thus increasing

\footnotetext{
Correspondence concerning this article should be addressed to J. Pauly at
} jerome.pauly@univ-pau.fr.

(C) 2005 American Institute of Chemical Engineers transportation costs and reducing productivity — and often leads to the plugging of pipelines requiring mechanical pigging to clean the pipe and restore transport. This problem is responsible for costs and losses in productivity that are estimated in billions of dollars per year in the oil industry. The situation has become increasingly worse, with the ongoing trend to deep water developments, and a predictive model that could help flow assurance teams on the design and preventive maintenance of pipelines would be a most valuable tool. ${ }^{1}$ Most of the experimental and simulation investigations carried out on the precipitation of heavy paraffins were undertaken at atmospheric pressure for dead oils, fuels, ${ }^{2-10}$ and synthetic systems. ${ }^{11-16}$ Nevertheless, investigation of paraffin precipitation 
cannot be dissociated from the pressure effect because the gases in the live oil can have a considerable influence on the wax appearance temperature (WAT) by decreasing it as much as $15 \mathrm{~K}$ between atmospheric and saturation conditions, ${ }^{17,18}$ and produce an increase of the WAT of about $2 \mathrm{~K}$ for each 10 MPa above that point. Thus the pressure effect also has to be well described by a thermodynamic model to predict the risk of waxy solid deposits during production.

For this purpose, some models, which rest on the restrictive and physically unsound assumption of multiple pure solid phases, have been proposed ${ }^{19,20}$ to describe the wax precipitation in condensate gases and crude oils. Another model, based on the formation of a solid solution that uses a cubic equation of state (EOS) with the LCVM (linear combination of HuronVial and Michelsen) mixing rule for the description of the fluid phases $^{21}$ and the Wilson equation ${ }^{22}$ for describing the solid solution nonideality, has been developed and was shown to accurately predict the onset of crystallization as well as the amount of solid precipitate as a function of temperature and pressure in both synthetic systems ${ }^{1}$ and crude oils. ${ }^{23}$ Unfortunately, this last model, which calculates the fluid-solid-phase equilibria with good accuracy, is difficult to implement in multiflash algorithms used in the petroleum industry because of the use of the $G^{\mathrm{E}}$ mixing rule as well as group contribution. This reduces the interest of the model, in spite of its success and reliability, for regular application in the petroleum industry.

To overcome these shortcomings, a model with the same predictive capacity - but easier to implement in conventional petroleum simulators- has been developed in this work. In this perspective the $\mathrm{G}^{\mathrm{E}}$ mixing rule has been replaced by the quadratic mixing rule. This change of mixing rule, however, modifies the fluid-phase fugacity, leading to a shift of the calculated wax appearance conditions. To overcome this situation the solid fugacity was also changed to reatin the quality of the liquid-solid equilibrium description by introducing a new parameter, $\xi$, in the $G^{\mathrm{E}}$ model used for the description of the nonideality of the solid phase.

This new model can be used to predict either (1) the influence of pressure or the addition of gas when the WAT for the stock tank oil is known by fitting the new parameter $\xi$ to the experimental data or (2) the wax appearance conditions when just the oil composition is available. For this purpose a correlation between the $\xi$ parameter and the width of the heavy paraffin distribution has been developed from literature data.

\section{Model Development}

\section{Fluid phase modeling}

The estimation of liquid and vapor fugacity coefficients is carried out using the Peng-Robinson equation of state (PR EOS $)^{24}$ with coefficients calculated from the correlations proposed by Robinson et al. ${ }^{25}$ It was chosen from among the available cubic equations of state because it provides a good description of high-pressure vapor-liquid equilibria of asymmetric light/heavy hydrocarbons systems with only two parameters $a$ and $b$. With respect to the previously proposed model, ${ }^{1}$ the mixing rules were simplified and the classical mixing rules (quadratic for $a$ and linear for $b$ ) were used with interaction parameter $k_{i j}$, evaluated by the correlations proposed by Pan et al. ${ }^{17}$ The critical properties required in the evaluation of the
EOS parameters were obtained from the compilation of Reid et $a^{2}{ }^{26}$ for light gases, whereas the Twu correlation ${ }^{27}$ is used to predict the critical properties of the heavy components. Because the slope of the melting line with pressure is closely related to the volume change of solid-fluid-phase transition, the volume of each phase has to be properly evaluated by the model for a good prediction of the pressure influence on liquid-solid-phase equilibrium. To achieve this requirement, the volumetric properties calculated by the original PR EOS are corrected by using a volume translation introduced by Peneloux et al. ${ }^{28}$

$$
V=V^{\prime}+C_{i}
$$

where $V^{\prime}$ represents the volume calculated from the PR EOS and $C_{i}$ is a translation parameter estimated at atmospheric pressure. $^{29}$

\section{Solid-phase modeling}

The variation of the fugacity $f_{i}^{w}$ of a heavy paraffin in the waxy part with pressure during an isothermal process can be evaluated by integration of the partial molar volume $\overline{V_{i}{ }^{w}}$ between the atmospheric pressure $P_{0}$ and the pressure $P$

$$
\ln f_{i}^{w}(P)=\ln f_{i}^{w}\left(P_{0}\right)+\frac{1}{R T} \int_{P_{0}}^{P} \overline{V_{i}^{w}} d P
$$

Several X-ray diffraction experiments clearly show that the wax forming during the crystallization of crude oils is made up of one or several solid solutions, depending on the difference between the temperature of the system and the melting temperature of the wax. To simplify the modeling, the waxy part may be-with the introduction of a negligible error-considered as a single phase whose composition $x_{i}^{w}$ corresponds to the average of the actual solid phases. In accordance with X-ray measurements, ${ }^{30}$ this waxy solid phase is assumed as a nonideal orthorhombic solution. The fugacity of a paraffin $i$ in this hypothetical waxy phase, at the reference pressure $P_{0}$, is therefore evaluated in terms of activity coefficients $\gamma_{i}^{w}$

$$
f_{i}^{w}\left(P_{0}\right)=x_{i}^{w} \gamma_{i}^{w} f_{i}^{s_{0}}
$$

where $f_{i}^{S_{0}}$, which represents the fugacity of the pure solid at the same temperature and pressure $P_{0}$, is related to the pure subcooled liquid fugacity $f_{i}^{L_{0}}$ from the change of free energy between the pure solid and the liquid at temperature $T$ and reference pressure $P_{0} \cdot{ }^{31}$ Assuming the thermal contribution of the Gibbs energy variation is negligible, the change of Gibbs energy can be expressed by

$$
\ln \frac{f_{i}^{S_{0}}}{f_{i}^{L_{0}}}=-\frac{\Delta G^{S L_{0}}}{R T}=-\frac{\Delta H^{S L_{0}}}{R T}\left(1-\frac{T}{T^{S L_{0}}}\right)-\frac{\Delta H^{t r_{0}}}{R T}\left(1-\frac{T}{T^{t r_{0}}}\right)
$$

where $T^{S L_{0}}$ and $\Delta H^{S L_{0}}$ represent the fusion temperature and the enthalpy of fusion, respectively; $T^{t r_{0}}$ is the solid-solid transi- 
Table 1. Systems Investigated with the Parameter $\boldsymbol{\xi}$ Fitted to WAT at Atmospheric Pressure

\begin{tabular}{lcclc}
\hline System & $n$-Alkane Range & Pressure Range (MPa) & Original Name of the System & Reference \\
\hline 1-SM & $20-30$ & $0.1-50$ & Mixture A & 12 \\
2-SM & $18-30$ & $0.1-50$ & Mixture B & 12 \\
3-SM & $18-27$ & $0.1-50$ & Mixture C & 12 \\
4-F & up to 32 & $0.1-100$ & Diesel Fuel & 36 \\
5-CO & up to 60 & $0.1-10.84$ & Crude Oil HI 557 A-1 & 37 \\
6-CG & up to 32 & $0.1-40$ & North sea crude oil CGA & 23 \\
7-CG & up to 35 & $0.1-40$ & North sea crude oil CGB & 23 \\
\hline
\end{tabular}

tion temperature; and $\Delta H^{t r_{0}}$ is the enthalpy of solid-solid transition.

The atmospheric activity coefficient $\gamma_{i}^{w}$ in Eq. 3, which takes into account the deviation from ideal behavior, is calculated using the predictive Wilson equation, ${ }^{16,22}$ which provides a good description of the phase behavior of orthorhombic solid solutions ${ }^{11}$

$$
\ln \gamma_{i}=1-\ln \sum_{j} x_{j} \Lambda_{i j}-\sum_{k} \frac{x_{k} \Lambda_{k i}}{\sum_{j} x_{j} \Lambda_{k j}}
$$

with

$$
\Lambda_{i j}=\exp \left[-\frac{\lambda_{i j}-\lambda_{j i}}{R T}\right]
$$

where the interaction parameter $\lambda_{i i}$ between two identical molecules is estimated from the enthalpy of sublimation, $\Delta H^{S V_{0}}$, by

$$
\lambda_{i i}=-\frac{2}{Z}\left(\Delta H^{S V_{0}}-R T\right)
$$

Because the interaction between two molecules arises only on the contact surface between them, it has been considered in a previous work ${ }^{16}$ that the interaction parameter $\lambda_{i j}$ is equal to the interaction parameter $\lambda_{i i}$ between two shorter molecules.

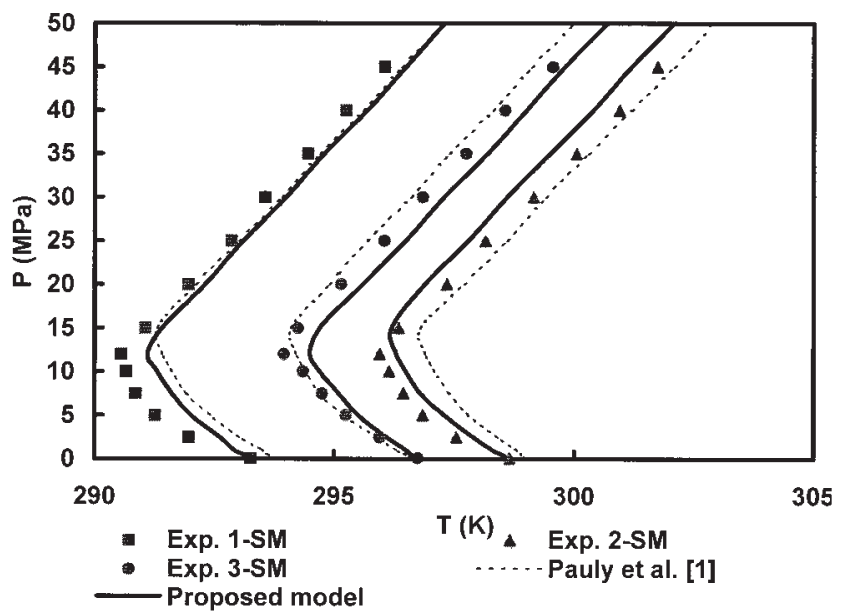

Figure 1. Wax appearance temperature (WAT) measured and calculated for 1-SM, 2-SM, and 3-SM under pressure.
However, this approach does not take into account the end effects, that is, the interactions arising at the chain ends. These can be accounted for by introducing a bending parameter

$$
\lambda_{i j}=\lambda_{i i}\left(1-\xi_{i j}\right)
$$

In this work, these bending parameters are replaced by a single average parameter $\xi$

$$
\lambda_{i j}=\lambda_{i i}(1-\xi)
$$

considered as an empirical parameter used to fit the model to the wax appearance measurements performed at atmospheric pressure.

The influence of pressure on the fugacity of waxes and thus on the fluid-wax equilibrium is taken into account by the Poynting term in Eq. 2. Evaluation of this term requires the knowledge of molar volume $\overline{V_{i}^{w}}$. As previously demonstrated, this quantity can be taken proportional to the liquid molar volume for pure components

$$
\overline{V_{i}^{S}}=V_{i}^{S_{0}}=\beta V_{i}^{L_{0}}
$$

with a proportionality coefficient $\beta$ assumed to be pressure independent and evaluated to 0.86 from the data reported by Shaerer et al. ${ }^{32}$ For mixtures, taking into account the excess volume of the solid phase, it was previously shown that the value of $\beta$ should be equal to $0.90 .{ }^{1}$ The Poynting correction can thus be rewritten in terms of pure liquid fugacity at pressure $P$ as

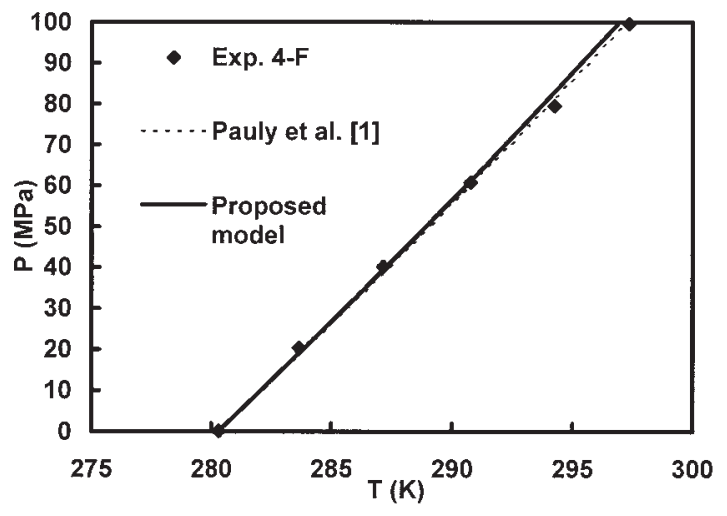

Figure 2. WAT measured and calculated from two approaches for 4-F under pressure. 


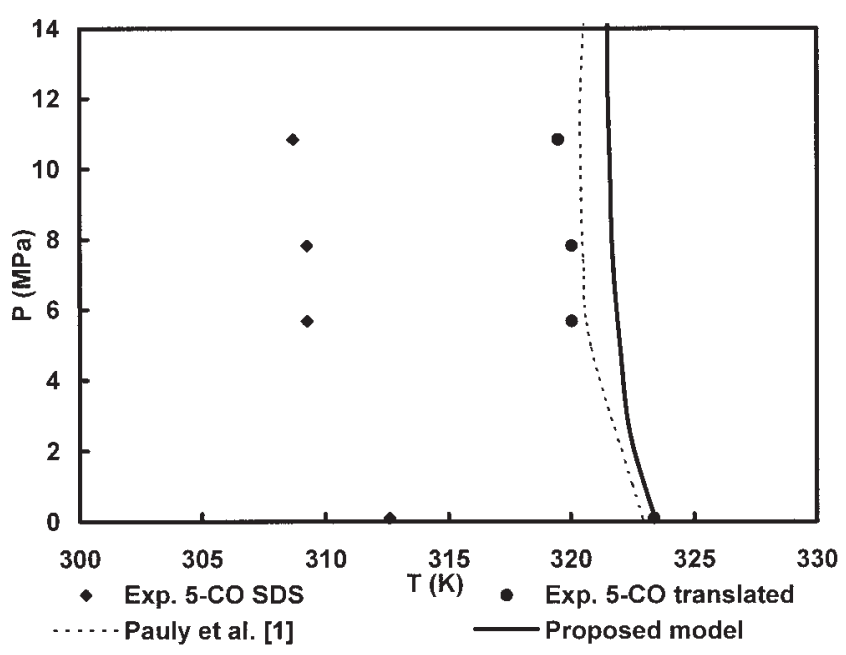

Figure 3. WAT measured and calculated from two approaches for 5-CO under pressure.

$$
\frac{1}{R T} \int_{P_{0}}^{P} \overline{V_{i}^{w}} d P=\frac{\beta}{R T} \int_{P_{0}}^{P} V_{i}^{L_{0}}=\beta \ln \frac{f_{i}^{L_{0}}(P)}{f_{i}^{L_{0}}\left(P_{0}\right)}
$$

Finally, by combining Eqs. 2, 3, 4, and 11, the expression of the solid fugacity in the solid phase at pressure $P$ is given by

$$
\begin{aligned}
f_{i}^{w}(P) & =x_{i}^{w} \gamma_{i}^{w}\left[f_{i}^{L_{0}}\left(P_{0}\right)\right]^{1-\beta}\left[f_{i}^{L_{0}}(P)\right]^{\beta} \\
& \times \exp \left[-\frac{\Delta H^{S L_{0}}}{R T}\left(1-\frac{T}{T^{S L_{0}}}\right)-\frac{\Delta H^{t r_{0}}}{R T}\left(1-\frac{T}{T^{r_{0}}}\right)\right]
\end{aligned}
$$

The major asset of this last expression is to separate the nonideality and the pressure influence on the fugacity of heavy paraffins in the waxy part. The evaluation of this property requires only the pure compound thermophysical properties $\left(T^{S L_{0}}, \Delta H^{S L_{0}}, T^{t r_{0}}, \Delta H^{t r_{0}}, \Delta H^{S V_{0}}, \beta\right)$, as well as the activity coefficient model at atmospheric pressure, the influence of pressure being estimated from the EOS used for the liquid phase. The parameter $\xi$ used for the determination of the activity coefficient in the solid phase can either be obtained from the correlation proposed below or used as a fitting parameter. The correlations proposed by Coutinho et al..$^{5}$ are used for the evaluation of melting and transition temperatures and enthalpies of the normal alkanes. Given that the waxy solid solution is assumed orthorhombic, the correlation for the odd paraffins is extended to the even paraffins and only this correlation is used for predicting the properties of the paraffins of the mixed heavy fraction.

\section{Fluid description}

The proposed model was tested against data for several fluids presented in the literature. These fluids were studied by various experimental techniques and were analytically characterized by using methods that have not been standardized. However, a description of petroleum fluids has been developed in this work to be used with the proposed model. It consists in separating the fluid into a light fraction that covers carbon dioxide, nitrogen, and all the hydrocarbons with less than 11 carbons and a heavy fraction $\mathrm{C}_{11+}$. The original description given by each author was kept for the light fraction, whereas the heavy fraction was split into the $n$-paraffin cut and the non- $n$-paraffin cut to differentiate between components able to precipitate and those that cannot. The $\mathrm{C}_{11+} n$-paraffin fraction is described by the detailed composition of normal paraffins from $\mathrm{C}_{11}$ because the WAT is substantially affected by the shape and size of the $n$-alkane distribution in the $\mathrm{C}_{11+}$ fraction. ${ }^{33-35}$ All the other nonparaffinic components including iso-paraffins, aromatics, and naphthenics compounds of the heavy fraction are gathered and described by a single pseudocomponent. This pseudocomponent is chosen to make the oil match the experimental average molecular weight of the fluid and its critical properties are estimated from the correlations proposed by Twu. ${ }^{27}$ When bubble points or dew points are available, the properties of this pseudocomponent can be tuned to match the liquid-vapor experimental curve.

\section{Results and Discussion}

\section{Atmospheric pressure fitting procedure}

Because the oil-wax phase behavior is easier to measure in stock tank oils than in live oils, it is advantageous for petroleum engineers to predict wax formation in oils in the presence of gas and under high pressure from measurements performed on the dead oil at atmospheric pressure. The proposed model has been designed from this perspective with a single parameter $(\xi)$, which can be tuned to a single experimental point. To determine the capacity of this approach, tests were performed on various systems. Experimental data of fluid-solid-phase equilibria under high pressure for crude oils or gas condensates are very scarce in the literature. Even for synthetic multicomponent mixtures data of this kind are limited to a few systems. The tests of the model were performed on eight different systems whose characteristics are summarized in Table 1. The first three systems (1-SM, 2-SM, 3-SM) are synthetic mixtures made up of $90 \%$ of the binary system methane + decane (with a molar fraction of methane of about $50 \%$ ) and $10 \%$ of a heavy fraction composed of various distributions of heavy normal paraffins centered around $\mathrm{C}_{22}$ and regularly decreasing. Daridon et al. ${ }^{12}$ reported liquid-solid and liquid-vapor phase-transition measurements up to $50 \mathrm{MPa}$ for these systems. The

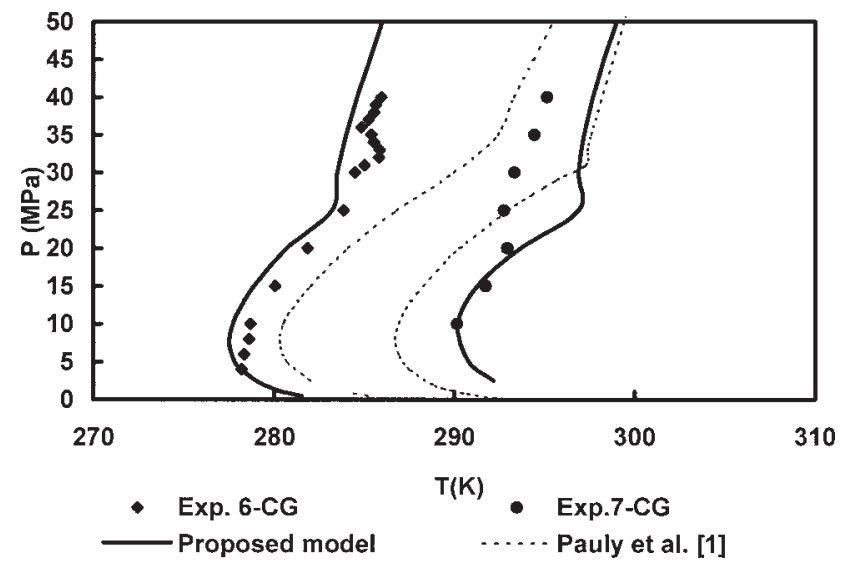

Figure 4. WAT measured and calculated from two approaches for 6-CG and 7-CG under pressure. 
Table 2. Mixtures Used for the Development of a New Correlation for $\boldsymbol{\xi}$

\begin{tabular}{|c|c|c|c|c|}
\hline System & $n$-Alkane Range & $\xi$-Fitted Parameter & Original Name of the System & Reference \\
\hline 8-SM & $18-30$ & 0.0410 & Mixture $\mathrm{B}^{\prime}$ & 11 \\
\hline 1-SM & $20-30$ & 0.0415 & Mixture A & 12 \\
\hline 2-SM & $18-30$ & 0.0430 & Mixture B & 12 \\
\hline 3-SM & $18-27$ & 0.0280 & Mixture C & 12 \\
\hline 9-SM & $18-35$ & 0.0570 & Mixture $\mathrm{B}+5$ & - \\
\hline $10-\mathrm{SM}$ & $18-40$ & 0.0740 & Mixture B +10 & - \\
\hline 11-SM & $18-45$ & 0.0860 & Mixture B +15 & - \\
\hline $12-\mathrm{SM}$ & $18-50$ & 0.1120 & Mixture B +20 & - \\
\hline $13-\mathrm{SM}$ & $18-55$ & 0.1370 & Mixture B +25 & - \\
\hline 14-SM & $18-60$ & 0.1590 & Mixture B + 30 & - \\
\hline $15-\mathrm{SM}$ & $18-65$ & 0.1815 & Mixture B +35 & - \\
\hline 16-SM & $18-70$ & 0.1840 & Mixture B +40 & - \\
\hline 17-SM & $18-80$ & 0.2055 & Mixture B +50 & - \\
\hline $18-\mathrm{SM}$ & $18-100$ & 0.2350 & Mixture B +70 & - \\
\hline $19-\mathrm{F}$ & $14-24$ & 0.0387 & Synthetic fuel SNC & 39 \\
\hline $20-\mathrm{F}$ & $10-25$ & 0.0487 & Diesel Fuel PB & 39 \\
\hline $21-\mathrm{F}$ & $10-17$ & 0.0197 & Fuel from shale 1 & 40 \\
\hline $22-\mathrm{F}$ & $11-17$ & 0.0143 & Fuel from shale 2 & 40 \\
\hline
\end{tabular}

fluid-solid-phase transitions were determined visually with an accuracy of $0.5 \mathrm{~K}$ by measuring the temperature of disappearance of the last crystal. For each mixture the parameter $\xi$ was fitted to match the experimental WAT at atmospheric pressure. The model was then used to calculate the wax appearance conditions up to $50 \mathrm{MPa}$. The results are plotted in Figure 1. It can be seen from this figure that the model correctly describes the phase envelope for these systems. At high pressures, the slope of the boundary between the liquid and the liquid-solid regions agrees with the experimental value and, at low pressures, the parabolic shape of the curve is also well represented by the model. These results show that when $\xi$ is fitted to atmospheric data, both the solubilization of methane and pressure effects are well described by the model in the case of mixtures whose composition is well known.

The fourth studied system is a diesel fuel (4-F) composed of paraffins ranging from $n-\mathrm{C}_{10}$ to $n-\mathrm{C}_{32}$ with a maximum on the distribution on $n-\mathrm{C}_{15}$. For this system, measurements of wax disappearance temperatures (WDTs) performed by cross-polarization microscopy (CPM) have been reported by Pauly et al. ${ }^{36}$ up to $100 \mathrm{MPa}$. Both the amount and the composition of the precipitated wax are also available for this fuel up to $50 \mathrm{MPa}$. As can be observed from Figure 2, the model also predicts well the influence of pressure on the WDT of this fuel that does not contain any gas.

The fifth investigated fluid (5-CO) is a crude oil from an offshore field in the Gulf of Mexico. For this fluid Hammami and Raines ${ }^{37}$ published WAT data on the live oil as well as on the stock tank oil. Measurements were performed in the stock tank oil by a laser-based solids-detection system (SDS) and by an atmospheric CPM technique, whereas for the live oil, the wax appearance was measured only by the SDS technique. As before, the model was fitted to the CPM WAT measured on the stock tank oil, which is considered by the authors themselves as the most reliable value. The high-pressure values calculated by the model were then plotted (see Figure 3) along with the experimental points measured by the SDS method. No agreement was found between the predictions of the model and the experimental points. The experimental WAT values are always more than $10 \mathrm{~K}$ lower than those predicted by the model. This deviation is mainly caused by the difference between the ex- perimental techniques used. Because of microscopy possibilities, the CPM method can detect wax crystals of $1 \mu \mathrm{m}$, whereas the SDS technique, which is based on light-scattering phenomena, detects the presence of waxes in a subsequent growth stage $(10 \mu \mathrm{m}) .^{38}$ This difference of sensitivity of the techniques leads to a difference in the WAT measured at atmospheric pressure of about $10 \mathrm{~K}$ for the stock tank oil. Assuming that the differences between the two techniques are repeatable and independent of pressure, the high-pressure SDS measurements were translated to correct them to the early stage of precipitation using the temperature difference measured on the stock tank oil. It can be observed from Figure 3 that the calculated and experimental data are in close agreement when the SDS measurements are corrected.

The two last fluids (6-CG and 7-CG) are two condensate gases from a high-temperature/high-pressure field in the North Sea for which Daridon et al. ${ }^{23}$ reported fluid-solid-phase transition above and below the dew point. For these fluids no measurements were performed on the condensate gases at atmospheric pressure. Therefore, the model was fitted to the lower pressure measurements ( $4 \mathrm{MPa})$. The value calculated by the model was then plotted (see Figure 4) along with the experimental points. The calculated values provide a description of the experimental data within experimental uncertainty.

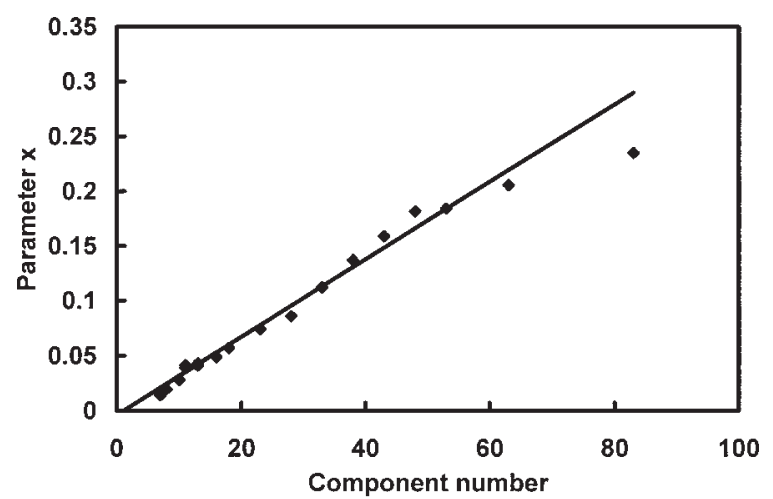

Figure 5. $\xi$ values vs. the number of components in the heavy fraction. 
Table 3. Predicted and Experimental WAT at Atmospheric Pressure for Real Systems*

\begin{tabular}{|c|c|c|c|c|c|}
\hline System & Original Name of the System & Paraffin Range & Experimental WAT (K) & Predicted WAT $(\mathrm{K})$ & Absolute Deviation \\
\hline $23-\mathrm{F}$ & Synthetic Fuel SNA & $12-22$ & 264.15 & 264.2 & 0.05 \\
\hline $24-\mathrm{F}$ & Synthetic Fuel SNB & $10-24$ & 271.15 & 271.3 & 0.15 \\
\hline $19-\mathrm{F}$ & Synthetic Fuel SNC & $14-24$ & 273.15 & 273.8 & 0.65 \\
\hline $25-\mathrm{F}$ & Diesel Fuel PA & $14-27$ & 284.15 & 281.5 & 2.65 \\
\hline $26-\mathrm{F}$ & Diesel Fuel PB & $10-25$ & 270.15 & 269.9 & 0.25 \\
\hline $27-\mathrm{F}$ & Diesel Fuel PC & $8-27$ & 268.15 & 269.7 & 1.55 \\
\hline $28-\mathrm{F}$ & Diesel Fuel PD & $10-27$ & 268.15 & 266.2 & 1.95 \\
\hline $29-\mathrm{F}$ & Diesel Fuel PE & $8-26$ & 266.15 & 265.6 & 0.55 \\
\hline $30-\mathrm{F}$ & Diesel Fuel PF & $9-27$ & 263.15 & 265.2 & 2.05 \\
\hline $31-\mathrm{F}$ & Diesel Fuel PG & $9-22$ & 261.15 & 261.5 & 0.35 \\
\hline $32-\mathrm{F}$ & Diesel Fuel PH & $9-25$ & 261.15 & 262.8 & 1.65 \\
\hline
\end{tabular}

*From Reddy. ${ }^{39}$

The model proposed here with the parameter $\xi$ fitted to atmospheric pressure data leads to a suitable description of fluid-solid-phase behavior. Both pressure and gas dissolution effects are well predicted by the model. Comparing the proposed approach with the predictive model developed by Pauly et al., ${ }^{1}$ it can be shown that there is no degradation of the pressure effect description on the WAT. The introduction of the parameter $\xi$ in the calculation of the activity coefficient in solid phase coupled with a cubic EOS using a classical quadratic mixing rule does not change the shape of the calculated solid-fluid transition curves. Moreover, it was found that, when this parameter is fitted to the experimental WAT at the lowest pressure, the difference between experimental and calculated temperatures does not exceed $3.5 \mathrm{~K}$ over the entire pressure range.

\section{Predictive procedure}

The procedure with the fitting parameter is advantageous when measurements can be performed on stock tank oils, although a model that could predict the conditions of wax appearance only from the knowledge of the fluid composition would be an important tool for the planning of field operation and transport facilities design. To confer a predictive nature to the proposed approach, a correlation for the parameter $\xi$ was developed. To build up this correlation, some experimental data of WAT performed at atmospheric pressure were taken from the literature. ${ }^{12,39,40}$ However, because the amount of data

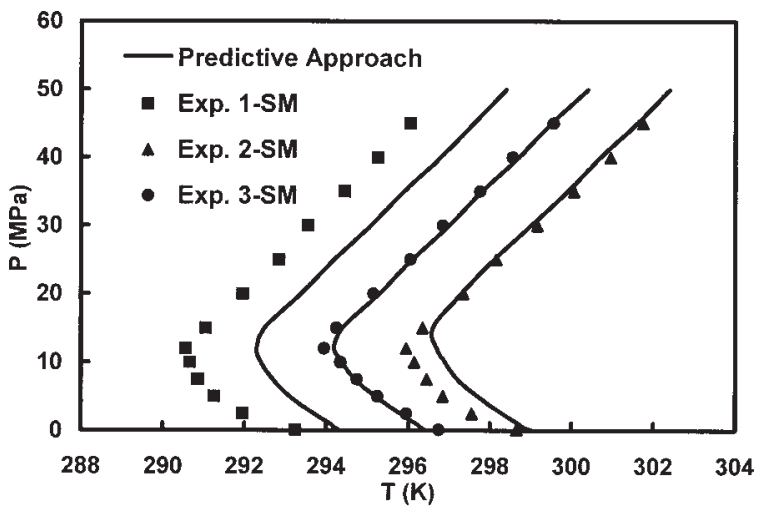

Figure 6. WAT measured and calculated for 1-SM, 2-SM, and 3-SM under pressure from the predictive proposed approach. available was not large enough and the composition of the investigated systems was too close, an artificial database was built. Starting from the mixture 2-SM, the composition of the artificial systems was built by gradually adding some heavy paraffins to reach, finally, a heavy fraction with 80 paraffins (mixtures from 9-SM to 18 -SM). The WAT values were then calculated from the predictive model of Pauly et al. ${ }^{1}$ because it has already been demonstrated that this approach provides very accurate results for synthetic systems similar to mixture B. ${ }^{1}$ Table 2 presents the different mixtures used for the development of the correlation. The range of the investigated mixtures in terms of carbon number in the heavy fraction ranges from 7 to 83 paraffins. For each of these mixtures the parameter $\xi$ was tuned to match the WAT of the database. The resulting values of $\xi$ were plotted as a function of the number of components in the heavy fraction, as shown in Figure 5. It can be observed that the dependency of $\xi$ on the number of components in the heavy fraction is practically linear. Moreover, the definition of the $\xi$ parameter (Eq. 9) requires that the correlation produces a value of $\xi$ equal to zero when the carbon number in the heavy fraction is one, corresponding to an ideal solid phase. The following expression was adopted to preserve this requirement

$$
\xi=a\left(N_{b}-1\right)
$$

where $a$ is equal to $3.537 \times 10^{-3}$ and $N_{b}$ represents the number of paraffin compounds on the $\mathrm{C}_{11+}$ fraction. For crude oils this number typically ranges between 20 and 50 .

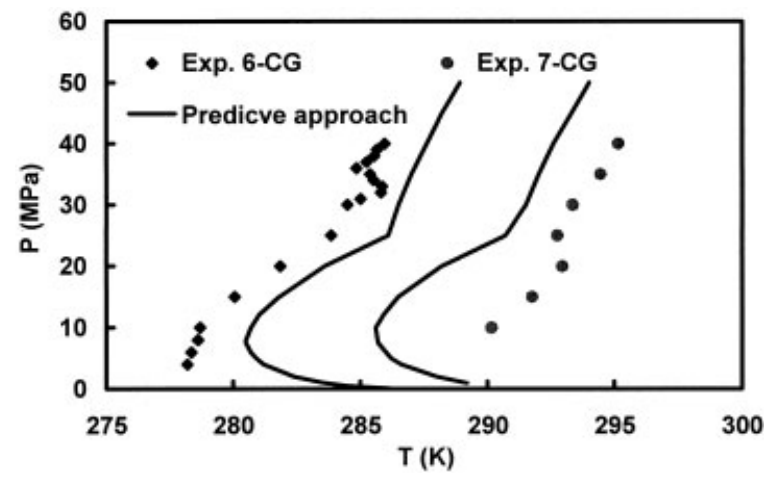

Figure 7. WAT measured and calculated for 6-CG and 7-CG under pressure from the predictive proposed approach. 


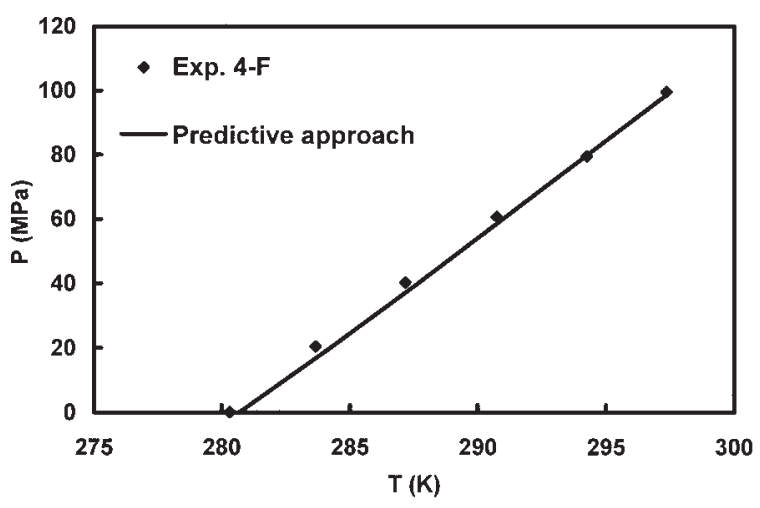

Figure 8. WAT measured and calculated for 4-F under pressure from the predictive proposed approach.

The predictive capacity of the model using the developed correlation of $\xi$ was also tested on the complex systems previously studied (Table 1), as well as on other atmospheric data for multiparaffinic systems ${ }^{39}$ that did not enter into development of the correlation.

In Table 3, a comparison between experimental and predicted WAT values at atmospheric pressure for all the systems studied by Reddy ${ }^{39}$ is presented. The absolute deviation does not exceed $2.65 \mathrm{~K}$ and the average deviation of about $1.1 \mathrm{~K}$ is well within the experimental uncertainty of the data for the different diesel fuels.

Figures 6 to 9 characterize the predictive calculations for the five different systems proposed in Table 1. The results are qualitatively and quantitatively in good agreement with experimental data, whereas the average absolute deviation between experimental and predicted wax appearance at high pressures is $<1.6 \mathrm{~K}$ and the effect of the pressure on WAT is well described both below and above the bubble point curve.

Finally, this approach was also used for the description of the amount of solid phase forming below the WAT. Figure 10 shows that the amount of solid precipitate vs. the pressure at one temperature for the mixture 8-SM (system 2-SM without

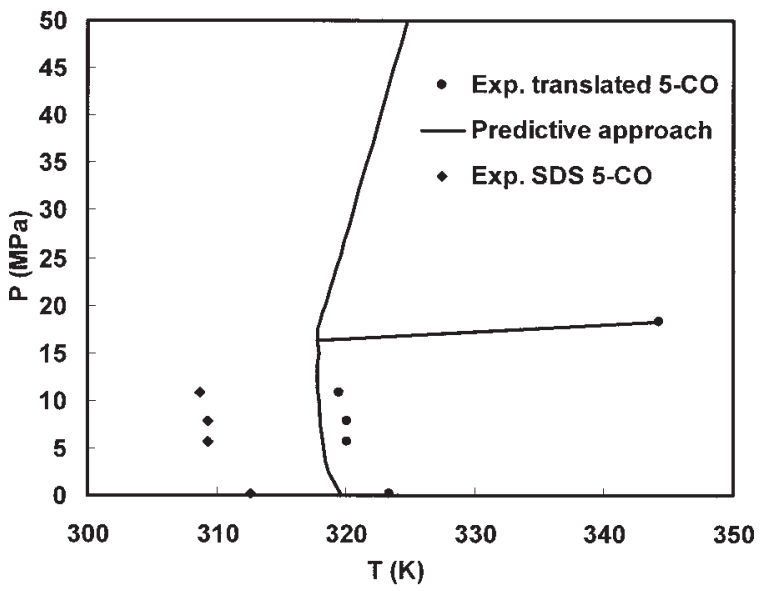

Figure 9. WAT measured and calculated for 5-CO under pressure from the predictive proposed approach.

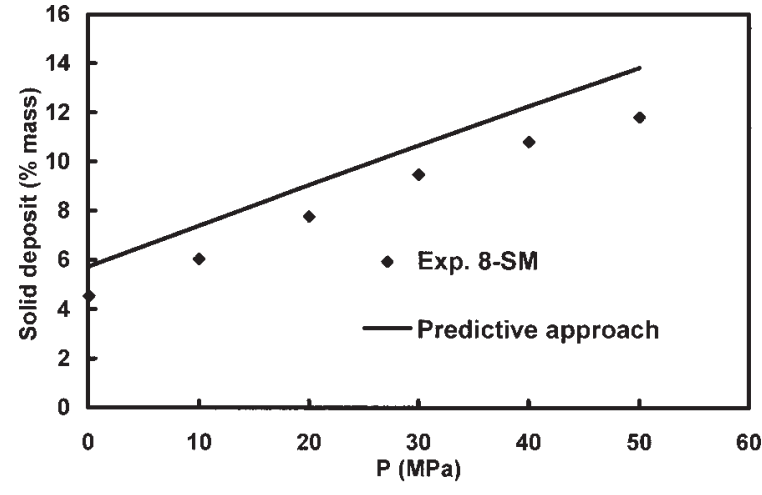

Figure 10. Comparison of experimental and calculated solid precipitate (in mass\%) for the system 8-SM vs. pressure.

gas $)^{41}$ is well described, particularly the effect of the pressure on solid precipitation. The same agreement between the calculation of the model and the experimental data was found (Figure 11) for the amount of wax precipitate in Fuel 4-F as a function of pressure. Moreover, Figures 12 and 13 show that the model leads to a good representation of the evolution of the waxy solid-phase composition with pressure.

\section{Conclusion}

A model to describe wax formation in petroleum fluids at high pressure is proposed. The results presented show that it can provide an excellent description of solid-fluid-phase boundaries for synthetic complex paraffinic mixtures as well as for real petroleum fluids. This is achieved by overcoming two shortcomings of previous approaches: using a cubic equation of state coupled with a simple mixing rule on a parameter: guaranteeing the continuity of the fugacities between the solid and fluid phases by modifying the solid activity coefficient value through a new corrective parameter $\xi$. A correlation relating $\xi$ to the number of components in the heavy fraction is proposed so that this new model can be applied predictively, using this correlation, or by tuning $\xi$ to some available experimental data. The proposed approach was successfully tested on a wide range of different real systems, allowing a good representation of the

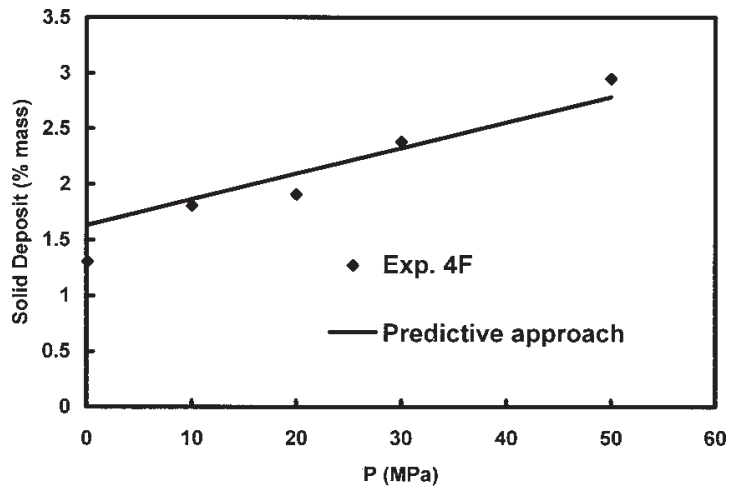

Figure 11. Comparison of experimental and calculated solid precipitate (in mass\%) for the system 4-F vs. pressure. 


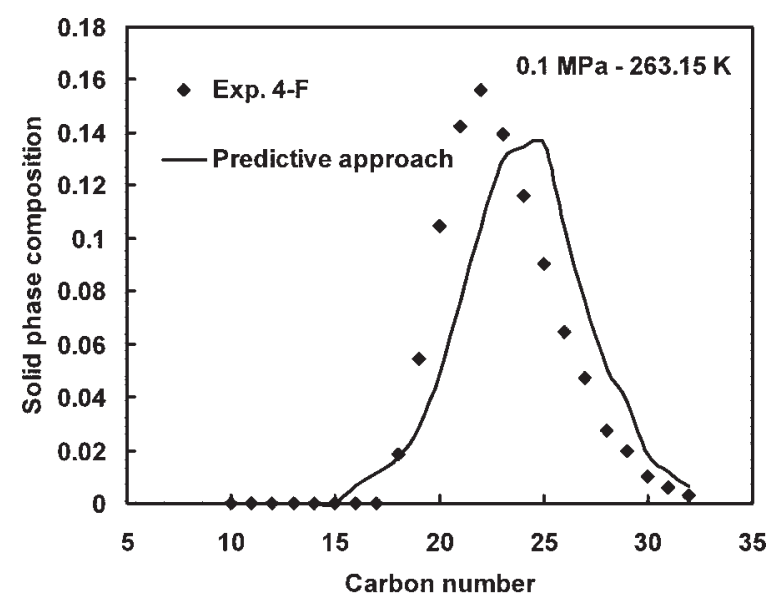

Figure 12. Comparison of the experimental and calculated solid-phase composition at 0.1 MPa for the system 4-F.

wax appearance temperature (WAT) under pressure and an accurate description of the solid precipitate under the WAT vs. the pressure.

\section{Notation}

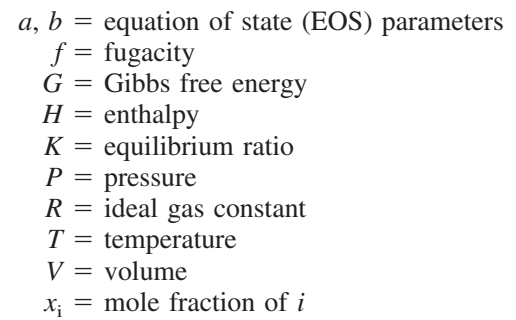

\section{Greek letters}

$\beta=$ volume change parameter

$\phi=$ fugacity coefficient

$\gamma=$ activity coefficient

$\lambda=$ interaction parameters of Wilson equation

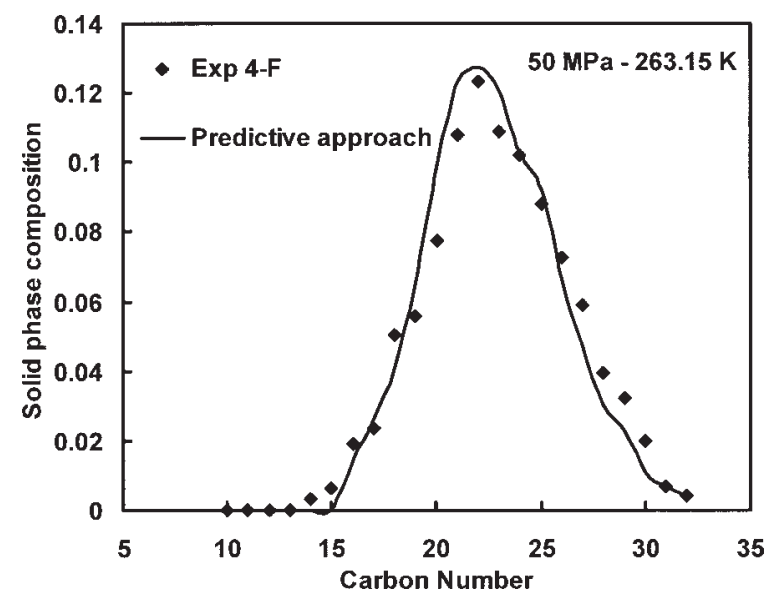

Figure 13. Comparison of the experimental and calculated solid-phase composition at $50 \mathrm{MPa}$ for the system 4-F.

$$
\xi=\text { corrective parameter }
$$

\section{Superscripts}

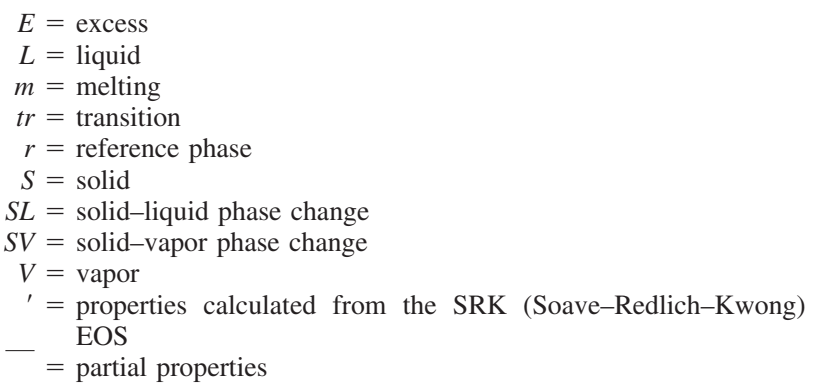

\section{Subscripts}

$$
\begin{aligned}
i & =\text { component } \\
n & =\text { carbon number } \\
o & =\text { pure component }
\end{aligned}
$$

\section{Literature Cited}

1. Pauly J, Daridon JL, Coutinho JAP, Lindeloff N, Andersen SI. Fluid Phase Equilib. 2000;167:145-159.

2. Pedersen WB, Hansen AB, Larsen E, Nielsen AB. Energy Fuels. 1991:5:908.

3. Moynihan CT, Mossadegh R, Bruce AJ. Fuel. 1984;63:378.

4. Van Winkle TL, Affens WA, Beal EJ, Mushrush GW, Hazlett RN, DeGuzman J. Fuel. 1987;66:890.

5. Coutinho JAP, Dauphin C, Daridon JL. Fuel. 2000;79:607-616.

6. Won KW. Fluid Phase Equilib. 1986;30:265.

7. Hansen JH, Fredenslund Aa, Pedersen KS, Rønningsen HP. AIChE J. 1988;34:1937.

8. Pedersen KS, Skovborg P, Rønningsen HP. Energy Fuels. 1991;5:924

9. Lira-Galena C, Firoozabadi A, Prausnitz JM. AIChE J. 1996;42:239.

10. Coutinho JAP. Energy Fuels. 2000;14:625-631.

11. Pauly J, Dauphin C, Daridon JL. Fluid Phase Equilib. 1998;149:191

12. Daridon JL, Xans P, Montel F. Fluid Phase Equilib. 1996;117:241.

13. Dauphin C, Daridon JL, Coutinho JAP, Baylère P, Potin-Gautier M. Fluid Phase Equilib. 1999;161:135-151.

14. Coutinho JAP, Ruffier-Meray V. Ind Eng Chem Res. 1997;36:49774983.

15. Coutinho JAP, Andersen SI, Stenby EH. Fluid Phase Equilib. 1995; 103:23.

16. Coutinho JAP, Knudsen K, Andersen SI, Stenby EH. Chem Eng Sci. 1996;51:3273.

17. Pan H, Firoozabadi A, Fotland P. SPE Prod Facil. 1993;Nov:250.

18. Rønningsen HP, Sømme BF, Pedersen KS. An improved thermodynamic model for wax precipitation: Experimental foundation and application to a field case. Proc. of 8th Int. BHR Group Conf.: Multiphase '97, Cannes, France, June 18-20; 1997.

19. Ungerer P. Fluid Phase Equilib. 1995;111:287-311.

20. Nichita DV, Goual L, Firoozabadi A. SPE Prod Facil. 2001;250-259.

21. Fredenslund Aa, Jones RL, Prausnitz JM. AIChE J. 1975;21:10861099.

22. Wilson GM. J Am Chem Soc. 1964;86:127-130.

23. Daridon JL, Pauly J, Coutinho JAP, Montel F. Energy Fuel. 2001;15: 730-735

24. Peng DY, Robinson DB. Ind Eng Chem Fundam. 1976;15:59-64.

25. Robinson DB, Peng DY. GPA Research Report 28. Tulsa, OK: Gas Processors Association; 1978.

26. Reid RC, Prausnitz JM, Poling BE. The Properties of Gases and Liquids. 4th Edition. New York, NY: McGraw-Hill; 1987.

27. Twu CH. Fluid Phase Equilib. 1984;16:137.

28. Peneloux A, Rauzy E, Frèze R. Fluid Phase Equilib. 1982;8:7.

29. Elbro, HS, Fredenslund Aa, Rasmussen P. Ind Eng Chem Res. 1191; 30:2576-2582.

30. Dirand M, Chevallier V, Provost E, Bouroukba M, Petitjean D. Fuel. 1998;77:1253-1260. 
31. Prausnitz JM. Molecular Thermodynamics of Fluid Phase Equilibria. Englewood Cliffs, NJ: Prentice-Hall; 1969.

32. Schaerer AA, Busso CJ, Smith AE, Skinner LB. J Am Chem Soc. 1986;77:2017.

33. Briard AJ, Bouroukba M, Petitjean D, Hubert N, Dirand M. J Chem Eng Data. 2003;48:497-513.

34. Dirand M, Bouroukba M, Chevallier V, Petitjean D, Behar E, RuffierMeray V. J Chem Eng Data. 2002;47:115-143.

35. Petitjean, D, Pierre M, Hubert N, Bouroukba M, Dirand M. Fuel. 2001;80:2007-2011.
36. Pauly J, Daridon JL, Sansot JM, Coutinho JAP. Fuel. 2003;82:595-601.

37. Hammami A, Raines M. SPE 38776, Edmonton, Alberta, Canada: DB Robinson Research Ltd. 1997.

38. Coutinho JAP, Daridon JL. J Petrol Sci Technol. in press 2004.

39. Reddy SR. Fuel. 1986;65:1647-1652.

40. Solash J, Hazlett RN, Hall JM, Nowack CJ. Fuel. 1978;57:521-528.

41. Daridon JL, Dauphin C. Meas Sci Technol. 1999;10:1-6.

Manuscript received May 20, 2003, and revision received Nov. 9, 2004. 